\title{
Evoked brain responses to auditory and visual stimuli of equal subjective magnitude
}

\author{
JAMES K. WALSH \\ Saint Louis University, St. Louis, Missouri 63103
}

\begin{abstract}
Evoked brain response (EBR) waveforms to auditory and visual stimuli of equal subjective magnitude were computed for three human observers. Amplitude-intensity functions for both modalities were described well by power functions. Power function exponents for auditory EBR functions were greater than for visual EBR functions. This finding corresponds with psychophysical data reported in the literature which indicate that loudness power functions grow faster than those for brightness. In addition, EBR waveforms computed to bimodal stimuli appear to be an algebraic summation of unimodal EBR waveforms. No sensory interaction is suggested by bimodal EBRs.
\end{abstract}

It is now well established that the amplitude of the evoked brain response (EBR) is related to changes in auditory (Davis \& Zerlin, 1966; Keidel \& Spreng, 1965; Schweitzer, 1977; Schweitzer \& Tepas, 1974; Tepas, Boxerman, \& Anch, 1972) and visual (Dinges \& Tepas, 1976; Kress, 1975; Shipley, Jones, Wayne, \& Fry, 1966; Tepas, Guiteras, \& Klingaman, 1974) stimulus intensity. Although these relationships are most frequently linear, their exact form is not clear. Often, logarithmic and power functions describe the data equally well. These linear EBR functions have been compared with psychophysical descriptions of the relation between stimulus intensity and sensation magnitude. Although both psychophysical and EBR relations to physical stimulus intensity can be described adequately by power functions, the exponents (i.e., slopes) of the EBR functions are typically smaller than those of comparable psychophysical functions (Botte, Bujas, \& Chochelle, 1975; Davis, Bowers, \& Hirsh, 1968; Keidel \& Spreng, 1965). Some investigators have concluded that these exponent differences suggest that any relationship between the EBR and sensation is quite indirect (Botte et al., 1975; Davis, 1974).

EBR functions similar to those typically measured psychophysically have been reported for several sensory phenomena. Dark adaptation (Fujimura, Tsuchida, Morita, \& Jacobsen, 1975; Klingaman, 1976), heterochromatic flicker photometry (Siegfried, Tepas, Sperling, \& Hiss, 1965), sequential blanking (Andreassi, Stern, \& Okamura, 1974), spectral sensitivity (Siegfried, 1971), and area-luminance effects (Kress, 1975) all produce EBR changes that correspond rather well to

This research was supported in part by NIOSH Grant 5 R01 OH 00395 to Donald I. Tepas. Requests for reprints should be sent to James K. Walsh, Sleep Disorders Center, Cincinnati General Hospital, Mont Reid Room 23, 234 Goodman Street, Cincinnati, Ohio 45267. psychophysical measurements. In some cases, relative changes in the EBR suggest that it may be just as sensitive as psychophysical determinations for the measurement of some sensory phenomena (Tepas, Walsh, \& Klingaman, Note 1). This seems especially true when EBR recording and stimulus control and presentation conditions rival the traditional high standards of most psychophysical research.

Stevens (1970) has negated the import of differences in exponents between electrophysiological and psychophysical power functions. As he points out, the consistency in relative exponent size should be the same for the EBR as for psychophysical determinations if the two measures have something in common. Thus, he argues in favor of a relationship between the EBR and sensation. His position is supported by the literature, in that the relative size of psychophysical power function exponents for different modalities usually approximates the relative size of EBR power function exponents. However, most studies collect EBRs in only one stimulus modality, and, therefore, EBR exponents for different modalities have been estimated using data from different subjects, different averaging procedures, and different recording strategies. Keidel and Spreng (1965) do report EBR functions for three sense modalities, but it is not clear whether the same subjects and methods were used for all modalities. Since there appears to be a great deal of interindividual variability in the exponents for both psychophysical and EBR functions, it is important to compare exponents derived from the same subject using similar testing methods. The present study examined EBR intensity functions to both auditory and visual stimuli. Comparable data from both modalities were collected from the same observers following psychophysical determinations of equal sensation magnitude.

Stevens (1970) has also suggested that stimuli of different modalities may evoke similar EBRs if the 
stimuli used are of equal subjective magnitude. Davis et al. (1968) report data which tentatively suggest that similar EBRs are produced by stimuli of equal subjective magnitude. Tepas, Schweitzer, Walsh, Dinges, and Flanagan (Note 2) also report data which may support this notion. The present study examines the proposal in finer detail, using auditory and visual stimuli.

With regard to sensory interaction, the results of previous reports of EBR measures are conflicting. Ciganek (1966) found little, if any, evidence for sensory interaction. Only a long-latency EBR deflection at around $230 \mathrm{msec}$ was significantly altered in a paired stimuli condition as compared to stimulation in a single modality. Davis, Osterhammel, Weir, and Gjerdingen (1972) report significant sensory interaction as indicated by differences in EBR amplitude. A test stimulus, in one modality presented $.5 \mathrm{sec}$ after a stimulus in a different modality, typically produced a smaller EBR than when it was presented alone. Shipley (1970) suggests that sensory interaction might be responsible for bimodal amplitudes being smaller than linear summation of the unimodal EBR amplitudes when recording from retarded children. Andreassi and Greco (1975) concluded that sensory interaction probably accounted for an increase in EBR amplitude to bimodal stimulation relative to unimodal stimulation in normals. Regan and Spekreijse (1977) report no EBR manifestation of the psychophysically suggested auditory and visual interaction. The bimodal EBR data of the present study were examined for evidence of sensory interaction.

In summary, the purposes of the present study were: (1) to examine auditory and visual EBR amplitude intensity functions for the same observers; (2) to determine if stimuli of different modalities matched for subjective magnitude result in similar EBRs; and (3) to study EBRs to bimodal stimuli for evidence of sensory interaction.

\section{METHOD}

\section{Subjects}

Three males, with a mean age of 26 years (range 23-29), volunteered to serve as observers. Each was a psychology student without apparent visual or auditory deficits. One observer had knowledge of the stimulus conditions, although he was not aware of the order of presentation used for his testing. The other two observers were naive in regard to stimulus conditions.

\footnotetext{
Apparatus

The stimulation and recording system has been described in detail by Tepas (1974). Auditory and visual stimulus presentation was initiated by Tektronix waveform and pulse generators. These generators triggered Grason-Stadler Series 1200 modules, which controlled the output of a Hewlitt-Packard function generator so that 50 -msec tone bursts of $1,000 \mathrm{~Hz}$ were presented $1 / \mathrm{sec}$ through matched TDH-39 earphones. The rise-fall times of these binaural tone bursts were $10 \mathrm{msec}$. Acoustical calibration was performed at the earphones using a Brüel and Kjaer Model 158 sound-level meter.

The Grason-Stadler modules also controlled a stepper motor
}

shutter in a two-channel Maxwellian view optical system. Monocular flashes were presented to the observer, who used a bite board bearing his dental impression as an aid in maintaining fixation. A tungsten ribbon filament lamp supplied light to both the target and surround channels. The target channel was used to present 50 -msec white flashes with rise-fall times of 7 and $9 \mathrm{msec}$, respectively. The second channel presented a constant circular surround of $400 \mathrm{~nm}$ with a half-bandwidth of $21 \mathrm{~nm}$. A constant current of $18 \mathrm{~A}$ was applied to the lamp from storage batteries, and flash luminance was varied by placing fixed-value Oriel neutral density filters (NDF) in the target channel. The white, circular flash target subtended a visual angle of $1.5 \mathrm{deg}$, and the maximum retinal illuminance available was $5.0 \log \mathrm{Td}$, as measured with a Macbeth illuminometer. The surround matched the target for brightness when approximately $3.2 \mathrm{log}$ units of NDF was placed in the target channel.

During bimodal stimulus presentation conditions, flashes and tone bursts had identical onset and offset times. This was determined by simultaneously averaging the voltage produced by a photocell placed in the position of the observer's eye and the auditory signal, using the LAB-8 computer system (Tepas, 1974).

The electroencephalogram (EEG) was recorded from a bipolar electrode configuration. Grass E5s electrodes were fastened to the scalp with colodion at $\mathrm{Cz}$ and $\mathrm{Oz}$ of the $10-20$ system. A $16-\mathrm{mm}$ Beckman bipotential electrode attached to the forehead served as ground. The EEG was amplified and charted by a Grass Model 7 polygraph equipped with a Model 7P5A preamplifier, and the amplified EEG potentials were fed into the AXO8 analog input of the LAB-8 computer system. Bandpass filters of the amplifiers were set at .15 and $500 \mathrm{~Hz}$.

The EEG was averaged by the Digital Equipment Corporation LAB-8 computer system, using the advanced averager program (DEC-LB-U18C-PB), for $500 \mathrm{msec}$ following stimulus onset. The averaging resolution was 1 point/msec. Prior to the start and at the completion of each session, the electrophysiological recording system was calibrated by averaging the square-wave output of a Grass stimulator, attenuated to $10 \mu \mathrm{V}$.

\section{Procedure}

Each observer participated in three training sessions to become familiar with the brightness-loudness matching task. A training session consisted of 20 psychophysical trials which were identical to those used in experimental sessions. Following training, observers were tested in 14 experimental sessions in which both psychophysical and electrophysiological data were collected. Intersession intervals were irregular and ranged from a minimum of $20 \mathrm{~h}$ to a maximum of 17 days. The median intersession interval was 3.5 days. Each session was a replication of the previous session in that the same number of psychophysical and electrophysiological trials were administered.

A psychophysical trial consisted of a series of flash-tone pairs presented 1/sec in blocks of 10 . After each block of 10 pairs, the observer made a match-no-match response via a hand-held signal button. At that time, tone intensity was either increased or decreased by $2 \mathrm{~dB}$ and the next block of 10 flash-tone pairs was presented. This procedure was continued until the observer had made at least one "match" response and two subsequent "nomatch" responses. The flash intensity was then changed, and a new trial was begun. In all cases, the experimenter adjusted both the auditory and visual stimulus levels. A more detailed description of the psychophysical methodology is presented elsewhere (Walsh \& Browman, 1978).

The same 10 flash intensities were used in each session. Intensities were equally spaced in .2 -log-unit steps. The maximum retinal illuminance used was $4.2 \mathrm{log} \mathrm{Td}$. For all 10 intensities, two ascending and two descending trials were completed in a session. The order in which the trials were administered varied from session to session in a random manner not disclosed to any of the observers. At the start of each session, the observer was adapted in the dark for $10 \mathrm{~min}$.

For each psychophysical trial, the initial auditory stimulus level 
was pseudorandomly selected in the following manner. The average match value of similar direction trials at a particular luminance from the previous session was noted. The initial value was then determined by randomly beginning from 1 to 9 steps ( 2 to $18 \mathrm{~dB}$ ) above or below that value.

Trials in which it was unlikely that a match would be made, based on previous matches were inserted randomly as control trials. In addition, auditory intensities were frequently repeated within a psychophysical trial as a further control. These control trials clearly resulted in observer responses confirming that proper care had been taken to eliminate possible observer bias or other factors which might confound the results of the psychophysical determinations.

Following the collection of the psychophysical matching data, average EEG samples were computed to five flash intensities, five tone intensities, and five flash-tone pairs. Odd-number sessions used .8, 1.2, 1.6, 2.0, and $2.4 \log$ units of NDF as the visual intensities for EEG averaging. Even-numbered sessions used 1.0, $1.4,1.8,2.2$, and $2.6 \mathrm{log}$ units of NDF. Auditory stimulus intensities for EEG averaging were determined directly from the results of the psychophysical matching data. For each session, linear regression functions were fit to the psychophysical data and auditory EBR stimulus intensities were taken to be the predicted decibel value paired with each visual stimulus intensity. The predicted tone intensities associated with each flash level were used as EBR stimuli in the same sessions as the corresponding flash level. Paired flash-tone EBR stimuli consisted of a flash intensity and the corresponding predicted tone intensity for a given session. EBR stimulus modalities and levels were administered randomly within a session, using a different order for each session. During all electrophysiological trials, the observer assumed a similar position, using the bite board. Thus, trials without flash stimuli were conducted with the target channel of the optical system blocked to allow observers to fixate on a portion of the surround. For each electrophysiological trial, the EEG potentials following 100 stimulus presentations were summed. Following each trial, this summed EEG activity was dumped on paper tape for later analysis.

After the completion of 14 sessions for a single observer, appropriate averaged EEG samples were pooled across sessions to form EBRs using software developed by Tepas, Kress, and Klingaman (1975). This procedure resulted in a single visual EBR (VEBR) for each of the 10 flash intensities. Auditory EEG samples were summed across sessions according to the flash intensity with which they were matched psychophysically. This resulted in a single auditory EBR (AEBR) matched to each of the 10 flash intensities. EEG samples to flash-tone pairs were similarly summed to produce an EBR for each of the simultaneous auditory-visual stimulation conditions (AVEBR).

All EBRs were plotted on an X-Y recorder, and digital values associated with the 500 points for each waveform were printed on the Teletype. Prominent peaks were identified, and measurements of peak-to-peak amplitudes were made from these digital values. The peaks measured are labeled in Figure 1 using letters a-f. These labels have no particular significance other than identification. Amplitude measures reported here are the differences between these peaks. They are $a-b, c-d$, and e-f for visual, auditory, and bimodal waveforms, respectively. Previous studies from our laboratory have indicated that these peak-to-peak deflections of EBRs recorded from a $\mathrm{Cz}-\mathrm{Oz}$ configuration are reliably related to both auditory (Tepas et al., 1972) and visual (Tepas et al., 1974) stimulus intensity over moderate ranges.

In addition to these naturally evoked and recorded EBRs, EEG samples were added or subtracted to produce hypothetical EBRs. Dissected EBRs (dEBR) are the result of subtracting one natural EBR waveform from a second natural EBR waveform. Synthesized EBRs (sEBR) are the sum of two naturally occurring waveforms.

\section{RESULTS}

Psychophysical cross-modality matching functions fit by the method of least squares followed the power law for each session for all three observers. Average correlational coefficients of $.98, .98$, and .99 for the three observers indicate the goodness of fit of the power functions. There were no significant differences among the exponents of these functions for a single observer. More extensive discussion of the psychophysical data is presented elsewhere (Walsh \& Browman, 1978).

Figure 1 displays all EBR waveforms collected in this study for Observer 3. The peak-to-peak amplitude measures $a-b, c-d$, and e-f are shown, as well as corresponding measures on dissected and synthesized waveforms, da-db, dc-dd, and se-sf.

Figure 2 shows the relationship between EBR log amplitude and auditory and visual log stimulus intensity for one observer. Linear regression lines are fitted to the data using the method of least squares. The general characteristics displayed for this observer are representative of the data for all three observers. In each case, the relation between EBR $\log$ amplitude and $\log$ stimulus intensity of both modalities is described by a power function.

Correlation coefficients and exponents of AEBR and VEBR power functions for each observer are shown in Table 1. The mean correlation coefficients and exponents are also shown. The slope of the AEBR function is steeper than the VEBR slope for
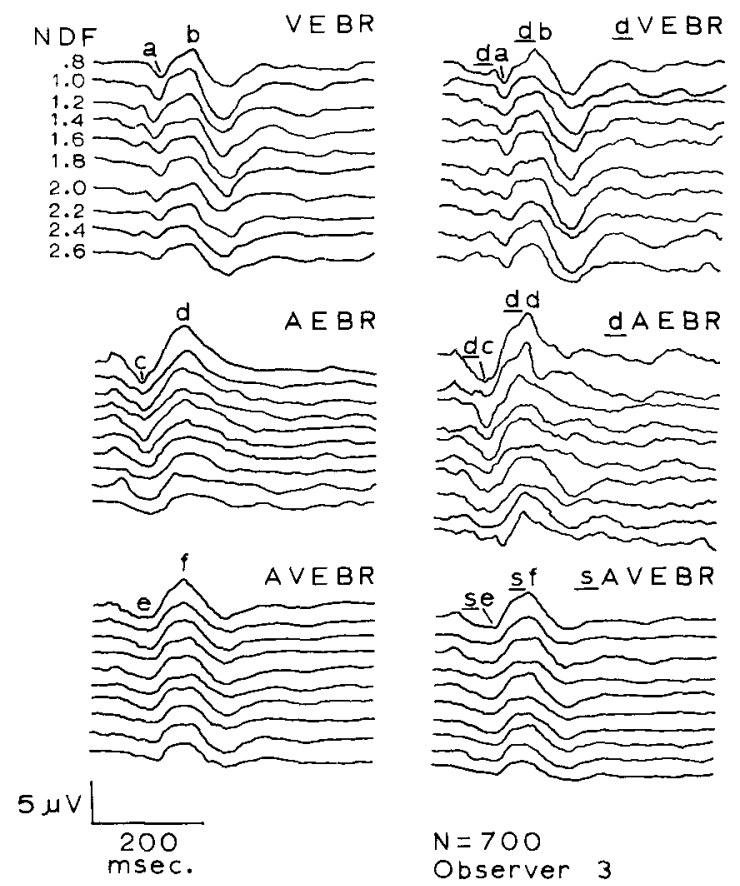

$N=700$

Observer 3

Figure 1. All EBR waveforms collected from Observer 3. The letters $a-b, c-d$, and e-f indicate the peak-to-peak amplitude measures reported in this study. Upward deflections indicate positivity at $\mathrm{Cz}$. Neutral density filtering is indicated to the left of the VEBR waveforms. The top EBR in each group is associated with the most intense stimulus, and the rest of the waveforms are in order of descending intensity. 


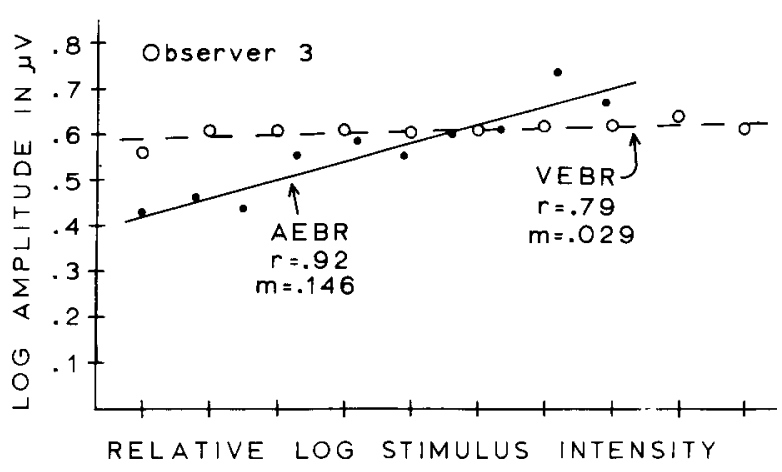

Figure 2. AEBR and VEBR log amplitude as a function of relative log stimulus intensity for a single observer. Hash marks on the abscissa represent .2-log-unit steps. The range of auditory stimulation for this observer was $1.4 \mathrm{log}$ units (87 to $59 \mathrm{~dB}$ SPL). The functions are arbitrarily anchored on the abscissa at the lowest intensity used for each modality. $M=$ exponent.

Table 1

Correlation Coefficients (r) and Power Function Exponents (m) for Naturally Recorded and Computer-Dissected EBR AmplitudeIntensity Functions for All Three Observers and Mean Data

\begin{tabular}{lcccccccc}
\hline & \multicolumn{2}{c}{ Observer 1 } & \multicolumn{2}{c}{ Observer 2 } & \multicolumn{2}{c}{ Observer 3 } & \multicolumn{2}{c}{ mean } \\
& $\mathrm{r}$ & $\mathrm{m}$ & $\mathrm{r}$ & $\mathrm{m}$ & $\mathrm{r}$ & $\mathrm{m}$ & $\mathrm{r}$ & $\mathrm{m}$ \\
\hline AEBR & .96 & .187 & .92 & .188 & .92 & .191 & .99 & .189 \\
VEBR & .83 & .071 & .91 & .174 & .79 & .029 & .85 & .091 \\
dAEBR & .84 & .205 & .78 & .150 & .89 & .172 & .84 & .242 \\
dVEBR & .11 & .015 & .45 & .076 & .67 & .029 & .44 & .040 \\
\hline Note- $r=.63, p<.05 ; r=.77, p<.01$
\end{tabular}

each observer, as well as for the mean data. The difference between the slopes did not reach significance for Observer 2, although the difference was in the same direction.

Computer synthesis and dissection of EBR waveforms yield waveforms that are remarkably similar to naturally recorded EBRs. As Figure 1 shows, AVEBR and SAVEBR waveforms are similar. In addition, comparison of AEBRs with dAEBRs and VEBRs with dVEBRs indicates that natural and dissected waveforms are comparable in their basic features. Waveforms from all three observers suggest a simple algebraic summation process.

Amplitude measurement of dAEBR and dVEBR waveforms produced functions similar to naturally recorded EBR functions. Figure 3 shows log amplitude data for dAEBR and dVEBR waveforms as a function of $\log$ stimulus intensity of Observer 3. Power functions describe the data adequately, and the dAEBR slope is significantly greater than the dVEBR slope. The dAEBR amplitude-intensity function for each observer was extremely similar to the AEBR function. Within an observer, no slope difference occurred. Correlation coefficients for two observers' dVEBR amplitude-intensity functions did not reach significance, but showed the same general trend in slope as their VEBR data. The dVEBR function of Observer 3 did not differ in slope from his VEBR data. Correlation coefficients and exponents of dAEBR and dVEBR power functions for the three observers and the mean data are also displayed in Table 1.

The relation between AVEBR and sAVEBR amplitude and stimulus intensity was adequately described by a linear function for only one observer. However, there was a high degree of similarity between AVEBR and SAVEBR functions for each observer. Figure 4 displays the AVEBR and SAVEBR amplitude data for Observer 3. The data are plotted as a function of flash intensity. Despite the nonlinearity of the functions, the agreement between naturally recorded and computer synthesized EBR functions is good. The agreement between AVEBR and SAVEBR functions for the other two observers is similar.

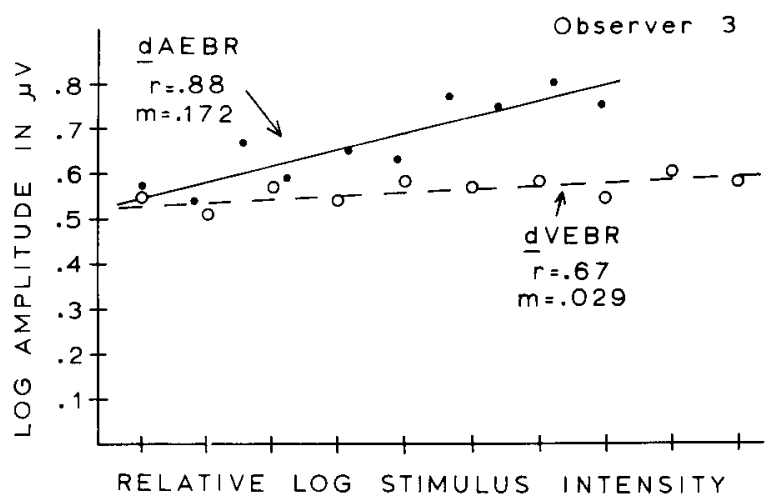

Figure 3. One observer's dAEBR and dVEBR log amplitude data as a function of relative log stimulus intensity. The symbol d refers to the computer dissection process used in this study. Dissected EBR waveforms are the result of subtracting one recorded EBR waveform from a second EBR waveform.

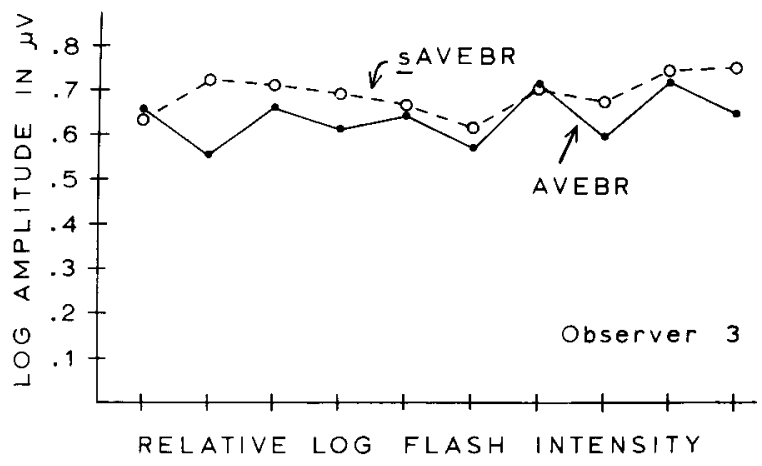

Figure 4. AVEBR and SAVEBR log amplitudes as a function of relative log flash intensity for Observer 3 . The symbol " $s$ " refers to the computer synthesis process used in this study. Synthesized EBR waveforms are the sum of two recorded waveforms. 


\section{DISCUSSION}

The data clearly indicate that EBR amplitude can be shown to vary systematically with the stimulus magnitude in both the auditory and visual sensory systems for individual subjects. The high degree of stimulus control and the selection of moderate stimulus intensities probably promoted the high correlation between stimulus magnitude and EBR amplitude.

Stimuli judged to be equally intense did not necessarily evoke equal sized EBRs. This finding clarifies tentative results previously reported by Davis et al. (1968) and Tepas et al. (1974). These studies did not use several intensities in each of the modalities matched for subjective magnitude, and, therefore, the results were partly dependent upon the intensities used. As can be seen in Figure 2, similar EBR amplitude was obtained for some, but not all, matched levels of stimulation. The present data depict the importance of studying ranges of stimulus intensity when examining nervous system function.

The AEBR amplitude-intensity function exponent (i.e., slope) is larger than the VEBR exponent for each observer. Since psychophysical measures also produce auditory intensity functions with higher exponents than do visual functions, these findings support the notion that psychophysical and EBR measures reflect similar neural processes (Stevens, 1970). A demonstration that the EBR slopes of other modalities are consistent, in a relative manner, with psychophysical exponents would provide more support for this hypothesis.

Stevens (1959) has shown that the exponent of a cross-modality matching function is predictable from the ratio of the single modality exponents derived in magnitude-estimation studies. If the EBR and magnitude estimation measurements reflect similar sensory processing, the ratio of the two unimodal EBR function exponents might have the same relative value as the cross-modality exponents. That is, observers with high psychophysical cross-modality exponents should have a relatively high ratio between the EBR function exponents. This speculation held true in the present study. The cross-modality exponents for the three observers were $.77, .83$, and 1.09 , and the ratio of the AEBR and VEBR function exponents for the three observers were $.15, .38$, and .93 , respectively. Relative to each other, each observer's crossmodality matching exponent held the same rank order as did the ratio of their AEBR and VEBR exponents. This is not to say that EBR function exponents can be used to predict absolute cross-modality matching exponent value, but this is another instance in which relative EBR measures match fairly well with comparable psychophysical determinations.

In the present study, cross-modality matching judgments were made using simultaneous flash-tone presentations, while some EBRs were collected to unimodal stimulation. If a sensory interaction occurred in the bimodal situation, comparison of unimodal EBR data using psychophysical matches made in the bimodal situation might not be justified. However, computer synthesis and dissection of EBR waveforms strongly suggest that bimodal EBR waveforms are the result of simple algebraic summation of unimodal EBR waveforms. Figure 1 shows that synthesized and dissected waveforms contain most of the basic properties of analogous naturally occurring waveforms. In addition, the relation of EBR amplitude to stimulus intensity for computer-constructed EBRs is amazingly similar to the naturally occurring relationship in many instances. AVEBR and sAVEBR functions are similar for all three observers, as are AEBR and dAEBR data. The failure of dVEBR data to mirror VEBR functions in all cases perhaps is related to the additional variability in the bimodal stimulation situation and computation of dissected waveforms. The lack of smoothness of dAEBR and dVEBR waveforms does suggest a poorer signal-tonoise ratio for dissected EBRs than for the VEBR and AEBR conditions. The findings that EBRs to bimodal stimuli seem to be the simple algebraic sum of two unimodal EBRs suggest that little, if any, sensory interaction occurred. The notion of simple algebraic summation of waveforms has been suggested previously (Schweitzer, 1977; Tepas et al., 1974; Tepas \& Walsh, Note 3). Thus, linear amplitudeintensity relations to bimodal stimulation should not, necessarily, be expected. Inter- and intraindividual differences in the latency and form of EBR deflections due to modality and stimulation level may involve cancellation and/or compounding of one deflection by other deflections. Because of this, one must approach with caution those studies which report EBR evidence for sensory interaction.

The use of a bipolar electrode configuration and a single amplification system minimized possible within-session variability in recordings due to changes in electrode resistance and/or amplifier response characteristics. This was extremely important, since recording sessions were at least $1 \mathrm{~h}$ in duration and electrodes were in place for about $3 \mathrm{~h}$. Nevertheless, bipolar recordings do not allow the identification of topographically related neural systems. Therefore, no specific statement about possible neural generators can be made for the present data. Additionally, it is possible that evidence for sensory interaction may not have been detected because the bipolar recording system may cancel potentials common to both $\mathrm{Cz}$ and Oz. Previous reports that EBR data do reflect sensory interaction have involved both bipolar (Ciganek, 1966; Shipley, 1970) and monopolar tech- 
niques (Andreassi \& Greco, 1975; Davis et al., 1972); therefore, it is unclear whether use of monopolar recordings would have produced evidence for sensory interaction in the present study.

Clearly, the EBR deflections measured and reported here are strongly related to sensory factors. This does not mean that these deflections are independent of attentional, motivational, or other psychological influences in all cases. However, the data do suggest that such factors can be minimized by using trained subjects, strict stimulus control, and large sample sizes, in order to study the relation between sensory dimension and the EBR. In addition, the apparent algebraic summation of EBR waveforms supports the suggestion of previous investigators (Goff, Allison, \& Vaughan, 1978) that different neural generators are involved in the production of the AEBR and VEBR deflections measured in the present study.

Future investigations of the EBR and its relation to psychophysical measures should examine other sense modalities such as the somatosensory or vibrotactal systems of the same subjects. Exponents for EBR intensity functions in these modalities should have the same relative value, when compared to other modalities, as do psychophysically determined exponents, providing that experimental conditions are rigidly controlled.

Finally, it should be noted that the number of studies which demonstrate good agreement between EBR and psychophysical estimates of sensory processing is growing. The continuing development of techniques and strategies for the collection of EBR data most probably has contributed to this increased agreement. The collection of both psychophysical and EBR data in the same session and the large number of EEG samples composing each EBR appear to be two strategies which most likely reduce the influence of random factors. In addition, precise control of stimulus parameters and the repeated testing of individual subjects may be important in demonstrating a relationship between EBR and psychophysical measurements.

\section{REFERENCE NOTES}

1. Tepas, D. I., Walsh, J. K., \& Klingaman, R. L. How sensitive are human evoked brain response estimates of sensation? Paper presented at the meeting of the Psychonomic Society, Denver, 1975.

2. Tepas, D. I., Schweitzer, P. K., Waish, J. K., Dinges, D. F., \& Flanagan, K. Human evoked brain responses to simultaneous auditory and visual stimulation. Paper presented at the meeting of the Psychonomic Society, Boston, 1974.

3. Tepas, D. I., \& Walsh, J. K. Additive property of human evoked response waveforms. Paper presented at the meeting of the Psychonomic Society, Washington, D.C., 1977.

\section{REFERENCES}

Andreassi, J. L., \& Greco, J. R. Effects of bisensory stimulation on reaction time and the evoked cortical potential. Physiological Psychology, 1975, 3, 189-194.

Andreassi, J. L., Stern, M., \& Okamura, H. Visual cortical evoked potentials as a function of intensity variations on sequential blanking. Psychophysiology, 1974, 11, 336-345.

Botte, M. C., Bujas, Z., \& Chochelle, R. Comparison between the growth of the averaged electroencephalic response and direct loudness estimations. Journal of the Acoustical Society of America, 1975, 58, 208-213.

Cigane K, L. Evoked potentials in man: Interaction of light and sound. Electroencephalography and Clinical Neurophysiology, $1966,21,28-33$.

DAvis, H. Relations of peripheral action potentials and cortical evoked potentials to the magnitude of sensation. In $\mathbf{H}$. $\mathbf{R}$. Moskowitz \& B. Scharf (Eds.), Sensation and Measurement. New York: Reidel, 1974.

Davis, H., Bowers, C., \& Hirsh, S. K. Relations of the human vertex potential to acoustic input: Loudness and masking. Journal of the Acoustical Society of America, 1968, 43, 431-438.

Davis, H., Osterhammel, P. A., Weir, C. C., \& Gjerdingen, D. B. Slow vertex potentials: Interactions among auditory, tactile, electric, and visual stimuli. Electroencephalography and Clinical Neurophysiology, 1972, 33, 537-545.

Davis, H., \& ZERLIN, S. Acoustical relations of the human vertex potential. Journal of the Acoustical Society of America, 1966, 39, 106-116.

Dinges, D. F., \& TePas, D. I. Luminance effects on visual evoked brain responses to flash onset and offset. Bulletin of the Psychonomic Society, 1976, 8, 105-108.

Fujimura, K., Tsuchida, Y., Morita, Y., \& Jacobsen, J. H. Application of visually evoked response near the threshold of vision to objective measurement of dark adaptation. American Journal of Ophthalmology, 1975, 79, 141-150.

Goff, W. R., Allison, T., \& Vaughan, H. G. The functional neuroanatomy of event related potentials. In E. Callaway, P. Tueting, \& S. Koslow (Eds.), Event-related brain potentials in man. New York: Academic Press, 1978.

Keidel, W. D., \& Spreng, M. Neurophysiological evidence for the Stevens' power function in man. Journal of the Acoustical Society of America, 1965, 38, 191-195.

Klingaman, R. L. The human visual cortical potentials and dark adaptation. Vision Research, 1976, 16, 1471-1477.

Kress, G. Area-luminance effects and the visual evoked brain response. Perception \& Psychophysics, 1975, 17, 37-42.

Regan, D., \& SpekreiJse, H. Auditory-visual interactions and the correspondence between perceived auditory space and perceived visual space. Perception, 1977, 6, 133-138.

Schweitzer, P. K. Auditory evoked brain responses: Comparison of $\mathrm{ON}$ and OFF responses at long and short durations. Perception \& Psychophysics, 1977, 22, 87-94.

Schweitze R, P. K., \& Tepas, D. I. Intensity effects of the auditory evoked brain response to stimulus onset and cessation. Perception \& Psychophysics, 1974, 16, 396-400.

SHIPLEY, T. Evoked brain potentials and sensory interaction in the retarded child. American Journal of Mental Deficiency, $1970,74,517-523$.

Shipley, T., Jones, R., WAyne, R., \& Fry, A. Intensity and the evoked occipitogram in man. Vision Research, 1966, 6, 657-667.

SiEgFrIEd, J. B. Spectral sensitivity of human visual evoked cortical potentials: A new method and a comparison with psychophysical data. Vision Research, 1971, 11, 405-418.

Siegraied, J. B., Tepas, D. I., Sperling, H. G., \& Hiss, R. H. Evoked brain potential correlates of psychophysical responses: Heterochromatic flicker photometry. Science, 1965, 149, 321-323. 
Stevens, S. S. Cross-modality validation of subjective scales for loudness, vibration and electric shock. Journal of Experimental Psychology, 1959, 57, 201-209.

Stevens, S. S. Neural events and the psychophysical law. Science, 1970, 170, 1043-1050.

TEPAS, D. I. Computer analysis of the electroencephalogram: Evoking, promoting, and provoking. Behavior Research Methods \& Instrumentation, 1974, 6, 95-110.

Tepas, D. I., Boxerman, L. A., \& Anch, A. M. Auditory evoked brain responses: Intensity functions from bipolar scalp recordings. Perception \& Psychophysics, 1972, 11, 21 7-221.

Tepas, D. I., Guiteras, V. L., \& Klingaman, R. L. Varia- bility of the human average evoked brain response to visual stimulation: A warning! Electroencephalography and Clinical Neurophysiology, 1974, 36, 533-537.

Tepas, D. I., Kress, G., \& KLingaman, R. L. APE: Average potential evaluation software for the LAB-8 system. Behavior Research Methods \& Instrumentation, 1975, 7, 120-124.

Walsh, J. K., \& Browman, C. P. Intra-individual consistency on a cross-modality matching task. Perception \& Psychophysics, 1978, 23, 210-214.

(Received for publication January 30, 1979; revision accepted September 21, 1979.) 